\title{
A Protocol for Space Charge Measurements in Full-size HVDC Extruded Cables
}

Prepared by the IEEE DEIS HVDC Cable Systems Technical Committee

G. Mazzanti, Chair

Dept. of El. Energy Engin. Information

University of Bologna

Viale Risorgimento 2

Bologna 40136, Italy

\section{N. Hozumi}

Dept. El. \& Electronic Inform. Engin.

Toyohashi University of Technology

1-1 Hibarigaoka, Tempaku-cho

Toyohashi, 441-8580 Japan

\section{F. Mauseth}

Norw. Univ. Science and Technology

Dept.of Electric Power Engineering

O.S. Bragstads Plass 2E

7491 Trondheim, Norway

\section{A. Tzimas}

ALSTOM Grid

Research \& Technology Centre

St Leonards Avenue

Stafford ST17 4LX, UK
G. Chen

Electronics and Computer Sciences

Southampton University

University Road, Highfield

Southampton SO17 1BJ, UK

J. Li

Dept. High Voltage \& Insulation Eng.

College of Electrical Eng.

Chongqing University

Chongqing 400044, China

\section{P. Morshuis}

Intelligent Electrical Power Grids

Delft University of Technology

Mekelweg 4

Delft 2628 CD, The Nederlands

\section{Kai Wu}

State Key Lab. of Electr. Insul. Power

Equip., Xi'an Jiaotong University

28 Xianning West Road

Xi'an 710049, China
J. C. Fothergill

Pro Vice-Chancellor (Research and Enterprise), City University London, Northampton Square

London EC1V 0HB, UK

\section{Marzinotto}

TERNA

Engineering Department

Viale Galbani 70

Roma 00156, Italy

C. Reed

USA Consultant

Scotia, New York 12302

USA

\begin{abstract}
This position paper, prepared by the IEEE DEIS HVDC Cable Systems Technical Committee, illustrates a protocol recommended for the measurement of space charges in full-size HVDC extruded cables during load cycle qualification tests (either prequalification load cycles or type test load cycles). The protocol accounts for the experimental practices of space charge measurements in the thick insulation of coaxial cables in terms of poling time, depolarization time, heating and cooling of specimens, as well as for the experience gained very recently from such kind of measurements performed in the framework of qualification tests relevant to ongoing HVDC cable system projects. The goal of the protocol is not checking the compliance with any maximum acceptable limit of either space charge or electric field. Rather, this protocol aims at assessing the variation of the electric field profile in the cable insulation wall during poling time at the beginning and at the end of load cycle qualification tests for full-size HVDC extruded cables. Indeed, in the design stage the electric field distributions are determined by the cable geometry and by temperature gradient in the insulation. Thus, the design is based on macroscopic parameters conductivity and permittivity and how they depend upon temperature. Any disturbance of the electric field due to space charge accumulation will only be revealed during space charge measurements either in as-manufactured state or in the aged state after load cycle qualification tests.
\end{abstract}

Index Terms - HVDC insulation, power cables, power cable testing, space charge. 


\section{INTRODUCTION}

HIGH Voltage Direct Current (HVDC) power transmission is becoming more and more attractive over High Voltage Alternating Current (HVAC) transmission, especially for bulk power delivery over long distances. Furthermore HVDC cables are the only option in certain circumstances, e.g. for long subsea links [1].

The traditional insulation for HVDC cables is oil-paper, particularly of the Mass Impregnated Non-Draining (MIND or MI) type, for which there is considerable service experience at voltage and (system) power ratings up to $500 \mathrm{kV}$ and 1000 MW, respectively [1-3]. Turning to HVDC cables with extruded insulation, they are relatively new. Nevertheless, as pointed out in [4], "recently, the number of installed HVDC links using cables with extruded-polymer insulation has increased, since polymer insulated cables have several advantages ... The principal advantages are that no oil is used in the insulation, and the maximum permissible conductor temperature in normal operation is higher"; in addition, jointing is far simpler. Direct-current voltages up to $\pm 320 \mathrm{kV}$ and power ratings up to $1 \mathrm{GW}$ are commercially available [5-8]. Furthermore, in December 2012 the Hokkaido-Honshu HVDC extruded bipolar cable link was commissioned, featuring $600 \mathrm{MW}$ transmission capacity and $\pm 250 \mathrm{kV}$ rated voltage, the highest in the world at present for HVDC extruded cable interties. This HVDC extruded cable system is also the first one that can be used at $90^{\circ} \mathrm{C}$ under polarity reversal via Line Commutated Current Source Converters (LCC) $[1,4,9]$.

It is well known that extruded insulation for cables subject to high-voltage DC is affected by trapped space charge. For this reason, various non-destructive methods for investigating space charge in solid polymeric materials set up over the last decades (for a review see e.g. [10-12]) have been essential for the R\&D activities that eventually led to the realization of actual HVDC extruded cable systems. However, as pointed out in [13]: "[while] abundant data has been obtained from film and plaque samples ..... less attention has been paid to space charge dynamics in full sized cables presumably due to a limited range of experimental systems suitable for examining cables. Such investigations would ... be more relevant to practical situations as all the features specific to cable design, such as insulator/semicon interfaces, insulating material processing, and divergent electric field would be fully reflected in the space charge behaviour. They would also make it possible to investigate the effect of a temperature gradient in radial insulation on the space charge accumulation, and hence replicate the conditions experienced when the cable is loaded in service" (after [13]).

Of course, measuring space charge in full-size cables ${ }^{1}$ is not an easy task, due to inherent experimental difficulties (see Sections 2, 5) [11]. However, since the 1990s space charge

\footnotetext{
1 "Full-size" HVDC cables have the same design and cross-section as those to be installed in the field.
}

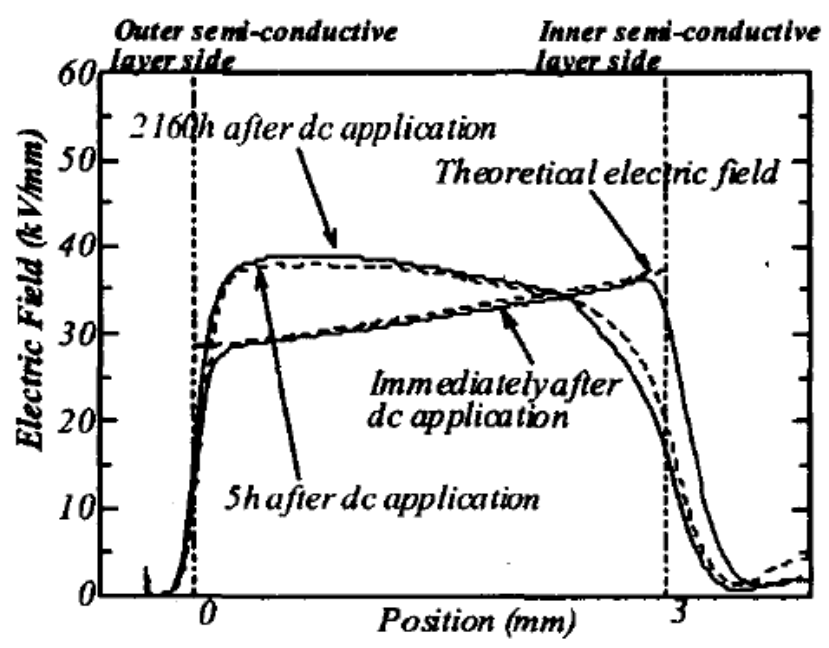

A)

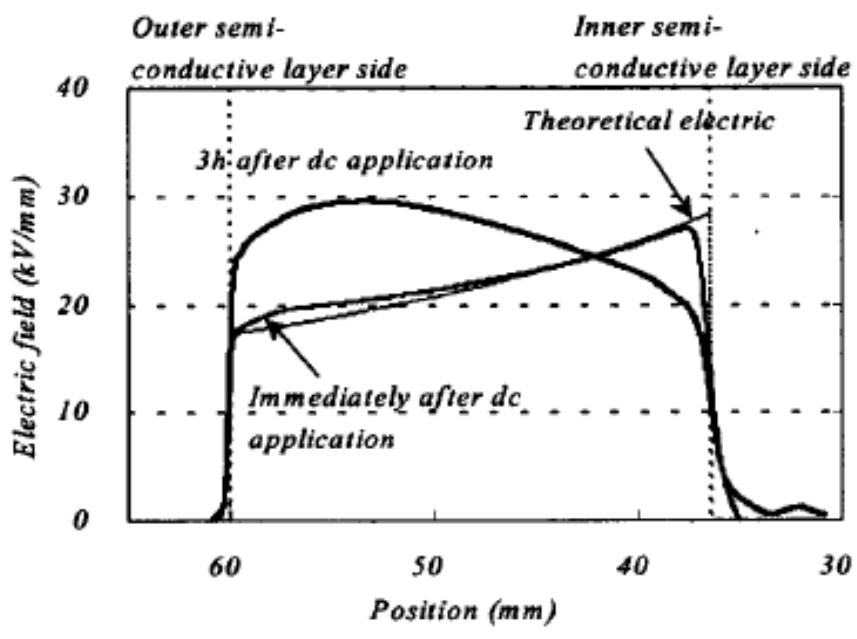

Figure 1. A) Electric field distribution in DC XLPE cable vs. applied voltage measured with the PEA method; insulation thickness $3 \mathrm{~mm}$ (after [16]); B) Electric field distribution of DC $\pm 500 \mathrm{kV}$ XLPE cable measured with the PEA method; insulation thickness $23 \mathrm{~mm}$ (after [16]).

measurements on large HVDC extruded cables with insulation thickness up to $23 \mathrm{~mm}$ have been reported to be satisfactorily performed by means of the Pulse-Electro-Acoustic (PEA) technique, see Figure 1 [2, 11, 14-17]. These results refer to measurements on full-size HVDC extruded cables performed as follows [14-17]:

1. on $\pm 250 \mathrm{kV}$ XLPE insulated cables, $12 \mathrm{~m}$ long including terminations, with $800 \mathrm{~mm}^{2}$ cross-section conductor and $20 \mathrm{~mm}$ thick insulation, poled at $\pm 500 \mathrm{kV}$ (for a maximum of $3 \mathrm{~h}$ on each polarity) under a severe temperature gradient (from $85{ }^{\circ} \mathrm{C}$ on conductor to $5-7{ }^{\circ} \mathrm{C}$ in open air where the cables were laid) $[14,15]$;

2. on a $\pm 500 \mathrm{kV}$ XLPE insulated cable with $3000 \mathrm{~mm}^{2}$ cross-section conductor and $23 \mathrm{~mm}$ thick insulation, poled at $\pm 500 \mathrm{kV}$ under temperature gradient $[16,17]$.

A few measurements on large cables with insulation thickness up to $5 \mathrm{~mm}$ have been reported to be satisfactorily performed by means of the Thermal Step Method (TSM) [18], that is accredited of being "applicable for thick insulators in the range of 2 to $20 \mathrm{~mm}$ " (after [10]). 
In addition, space charge measurements on full-size HVDC extruded cable loops using the PEA or the TSM techniques have been and are being performed in some laboratories worldwide for qualification tests ${ }^{2}$ for HVDC extruded cable link projects of major significance. The utilities that are in charge of these HVDC extruded cable link projects have included space charge measurements in the qualification procedure of cable system manufacturers following the above reasoning after [13]. Indeed, space charge dynamics in full-size cables to be installed in the field may differ from those in small(er) size insulation tested during R\&D activities. Thus, there is a stringent need to check that such differences do not "adversely affect the long term performance of the cable system" [19]. In fact, measuring space charges enables the evaluation of the real "Poissonian" electric field in the insulation wall $[11,13]$, that varies with laying conditions, load and ageing in a very peculiar way for full-size HVDC cables compared to smaller size insulation.

Therefore, the space charge measurements performed currently after prequalification (PQ) and type test (TT) load cycles in the laboratories of different manufacturers worldwide offer a very good opportunity for assessing the space charge and electric field profiles in the insulation wall of full-size HVDC extruded cables.

Unfortunately, neither a standard procedure, nor space charge limits have been universally agreed yet [20]. Hence these space charge measurements rely on project-dependent agreements between cable manufacturers and the customers. In this respect TERNA, the Italian Transmission System Operator (TSO), was the first in the world to include in its internal specifications the space charge measurement as a method to access the electric field distribution in the insulation wall of a DC extruded cable [21,22] during qualification tests (both PQ tests and TT) of DC extruded cables. Such method, applicable to both PEA and TSM techniques, has been successfully applied to ongoing projects.

As a first step of its activities started in year 2013, the IEEE DEIS Technical Committee "HVDC Cable Systems" has decided to focus on this issue, benefitting from some of the members having direct experience of these space charge measurements. The goal of the TC is to provide a more sound technical-scientific background that can serve as a reference for such measurements. For this reason the activity performed so far by the TC consists of proposing a recommended protocol for the measurement of space charges in full-size HVDC extruded cables during PQ and TT load cycles [21, 22]; this position paper illustrates and discusses such protocol.

The protocol aims at evaluating the electric field profile in the cable insulation wall, without checking the

\footnotetext{
${ }^{2}$ According to the prescriptions after CIGRÉ Technical Brochure 496 [19], qualification tests include: 1) prequalification (PQ) tests, based on a long sequence of 360 " 24 hours" heating-cooling cycles under constant DC voltage (referred to as "load cycles" from now on);2) type tests (TT), that encompass a shorter and simpler sequence of " $24 / 48$ hours" load cycles lasting 30 days.
}

compliance with any maximum acceptable limit of either space charge or electric field; indeed, as pointed out above, there is no universal agreement on limits for measured space charge or for the associated electric field [20]. On the contrary, this protocol focuses on charge and field dynamics during the measurement period, and particularly on how the electric field changes during the measurements, thereby monitoring such changes at selected stages of PQ and TT load cycles for full-size HVDC extruded cables. Indeed, in the design stage the electric field distributions are determined by the cable geometry and by the temperature gradient in the insulation. Thus, the design is based on the macroscopic parameters of conductivity and permittivity and their temperature dependence. Any disturbance of the electric field due to space charge accumulation will only be revealed during space charge measurements either in an as-manufactured state or in the aged state after load cycle qualification tests.

In Section 2 the space charge measurement methods chosen in the protocol, i.e. PEA and TSM - as they have resulted the best-suited for space charge measurements in large size HVDC extruded cables - are illustrated with reference to such cables. In Section 3 the limitations of the first proposal of the protocol are discussed. In Section 4, the protocol is described in detail for both the PEA and the TSM technique and for both PQ and TT load cycles. In Section 5 the main difficulties encountered in the measurements are discussed. Finally, some conclusions are drawn.

\section{MEASUREMENT METHODS IN THE RECOMMENDED PROTOCOL}

Measuring space charge in full-size cables is not an easy task, due to inherent experimental difficulties [11]. However, as pointed out in Section 1, space charge measurements on full-size cables have been satisfactorily performed since the 1990s by means of two techniques:

- the Pulse-Electro-Acoustic (PEA) method [10,11, 14-17];

- the Thermal Step Method (TSM) [10, 18].

For this reason, the recommended protocol considers these two space-charge measurement methods for space charge measurements on full-size HVDC cables. They are also considered as they have been or are being employed for space charge measurements on full-size HVDC extruded cables during pre-qualification and type tests of major HVDC projects. For the sake of completeness, the main details of these two methods are reported in this section.

It must be pointed out that also the Pressure Wave Pulse (PWP) method, especially when implemented according to the Laser Induced Pressure Pulse (LIPP) technique, has been considered for local space charge measurements on cables with insulation thicker than that of mini-cables (say, up to $\sim 3$ mm) [23-25], but -to the best of our knowledge - no measurements with this method have ever been performed on full-size cables. For this reason the PWP method is not considered here. 


\subsection{PULSE-ELECTRO ACOUSTIC (PEA) METHOD}

The principle of the PEA method is summarized in [11] by its inventor, Prof. Takada: "Consider a plate sample with thickness $d$ and space charge distribution $\rho(r)$. A ... pulsed electric field $e_{p}(t)$ is applied to the sample and induces a perturbation force on each charge. This force causes the charge to move slightly. This movement launches an acoustic wave that travels at the speed of sound $u_{S}$ and is proportional to the charge distribution in the sample. A piezoelectric transducer is used to detect the acoustic wave and transform the acoustic wave into an electric signal" (after [11]).

The PEA method was originally conceived for space charge measurements on thin samples between plane parallel electrodes [26], but later it was extended successfully to the coaxial cable geometry [27] using the system shown in Figure 2.A (after [2]): $a$ and $b$ are the inner and outer radii of the insulation, respectively; $\rho(r)$ is the volume space charge density at radius $r ; s, l$, and $c$ are the thickness of the outer semicon, the distance between the outer semicon and the piezoelectric transducer - a polyvinylidene (PVDF) film - and the position of the piezo device, respectively. $R$ and $C$ are respectively the resistance and capacitance of the PEA measurement system, $V$ is the voltage of the DC source, and $v_{p}(t)$ is the time-dependent applied voltage pulse. $v_{s}(t)$ is the voltage signal from the piezo device, whereby the space charge distribution is derived [2]. As made clear by Figure 2.A, the cable sample electrode has coaxial structure, thereby granting a close contact between the cable and the electrode; the PVDF film is carefully positioned between the detecting electrode and the absorber avoiding any air gaps to prevent acoustic wave reflection at the interface between electrode and transducer $[2,11]$. The detecting electrode also acts as an acoustic delay line to enable the small piezoelectric signal to be distinguished from the electrical noise induced by the voltage pulse.

For large size HVDC cables, if the pulse voltage were applied to the HV terminations, it would travel along the cable and - considering the characteristic impedance of the large cable - it would be distorted and/or reflected. Thus, the signal at the measuring point would not be a single pulse [11]. In [28] a measurement set-up is proposed for solving these problems (see Figure 2B, after [15]): the voltage pulses are applied between the measuring point (at the centre of an exposed section of the outer semicon) and ground. If the characteristic impedance of the cable is much lower than the impedance of the capacitance at the measuring point - in the frequency range of the voltage pulses - most of the voltage pulse is applied at that point (see Figure 2C). Typically, voltage pulses are a few $\mathrm{kV}$ high and some tens ns wide, and the PVDF film is $\approx 100 \mu \mathrm{m}$ thick. The signal is amplified within a shielded box that is electrically isolated (a HV pulse is applied there) and is eventually transferred to a digital oscilloscope with high sampling frequency via an optical fiber $[11,14,15,28]$.
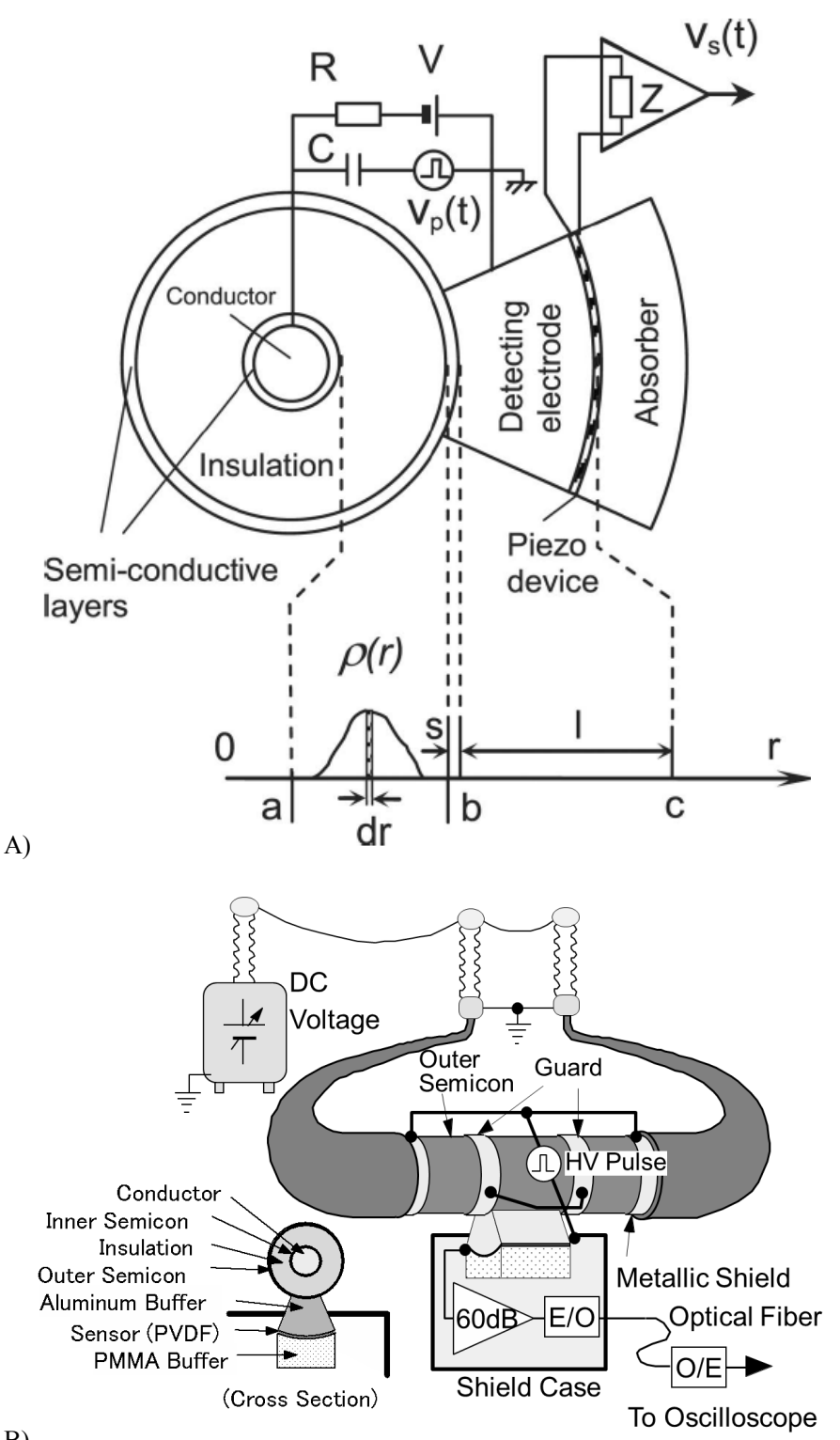

B)

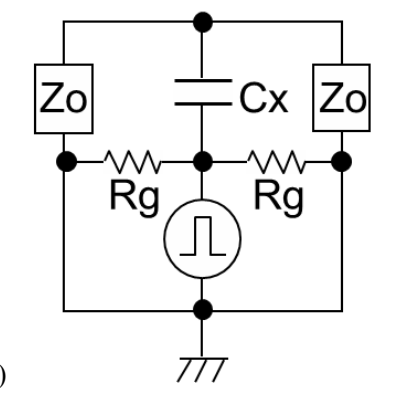

\section{Cx: Capacitance at the Measuring Point}

Zo: Characteristic Impedance of Cable

Rg: Resistance of the Exposed Semicon

Figure 2. A) Principle of the pulsed electro-acoustic (PEA) method for the measurement of space charge in cylindrical geometry. (after [2]). B) experimental setup for space charge measurement for a long cable with the PEA method (after [15]). C) equivalent circuit (after [11]).

The PEA system with curved ground electrode, transducer and acoustic absorber block - implemented and used by various R\&D teams in the world - has shown excellent performances on cable models of various sizes, even in the presence of multiple interfaces [29-33]. Other have 
successfully used a modified version of the PEA cell with curved ground electrode, transducer and absorber, with slightly different electrical connections from the HV pulse source to the outer semicon and the metallic shield of the cable (more details are omitted here, they can be found in [34]). However, a different configuration with a flat ground electrode - that can be easily applied to cables of different diameters - has also been proposed and employed satisfactorily for cable models with insulation thickness up to $\sim 6 \mathrm{~mm}[13,35]$.

Further details about the PEA measurement set-up for a full-size cable are outside the goal of this recommendation. They can be found, e.g., in [2, 11, 14-17, 34].

\subsection{THERMAL STEP METHOD (TSM)}

The TSM has also enabled space charge measurements on cables with thick insulation $[10,18]$. It relies on the application of a thermal step (TS) across an insulating system. Because of the thermal expansion of the dielectric, a thermal wave is created that travels through the insulation, whereby local space charge is temporarily displaced [36, 37]. This alters the induced charges at the electrodes, causing a current between the electrodes if short-circuited; the mathematical processing of this current provides the distribution of the electric field and charge density within the insulation wall. Thus, the electrodes are short-circuited through a current amplifier, connected to a PC that records and processes the current.

The TSM is suitable for any sample whose space-time temperature distribution can be computed. This is the case of power cables, for which the TS can be created by means of two alternative techniques, namely [18]:

- the inner heating technique (IHT), whereby the TS is generated through a strong current (several kA) circulating in the cable conductor. The conductor in short circuit works as the single-turn secondary of a heating transformer. After several seconds of heating, the conductor and the outer semi conductive layer are short circuited through a current amplifier. The IHT aims at following the evolution of the mean electrical state of the whole cable insulation and does not require any specific preparation before measurements;

- the outer cooling technique (OCT), whereby the TS is created through a cold liquid circulating within a radiator in contact with the sample (Figure 3 ). The OCT aims at a local analysis of the cable over small lengths ( $\sim 20$ to 40 $\mathrm{cm})$. In the proposed protocol this technique is chosen.

For measurements under DC voltage, the setup of Figure 3 has two main drawbacks: 1) the current amplifier must not be in contact with the high voltage; 2 ) if the current amplifier is placed between the sample subjected to $\mathrm{HV}$ and ground, the conduction and the polarization currents may mask the TS current. For this reason, the TSM in "double capacitor" configuration is used in practice, whereby a "compensation cable" (identical to the cable under measurement) is inserted by connecting the external semicon of the compensation cable

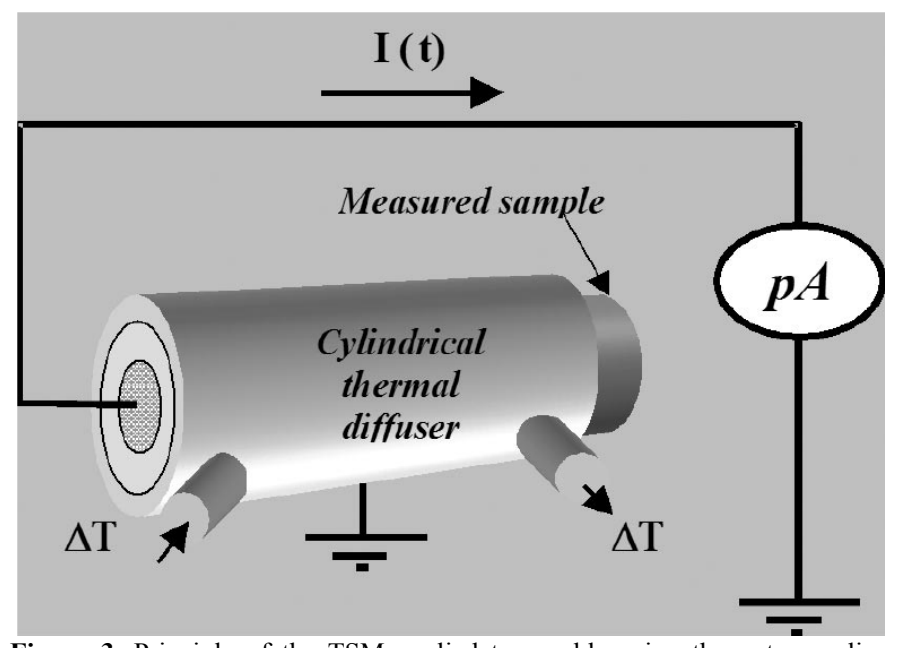

Figure 3. Principle of the TSM applied to a cable using the outer cooling technique in short-circuit conditions (after [18]).

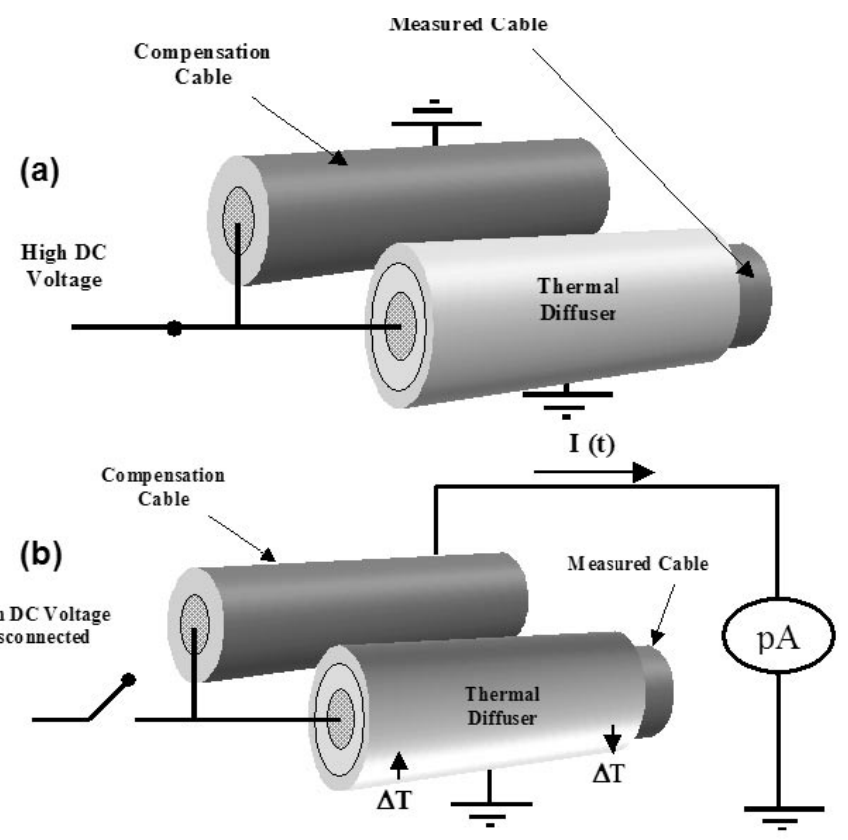

Figure 4. Under voltage TSM measurements on cables in double capacitor configuration. (a) the dc voltage is applied prior to the measurement, (b) the measurement is performed on the measured cable after disconnecting the $\mathrm{dc}$ source. (after [18]).

to a current amplifier and its conductor to that of the cable under measurement. Thus, the space charge measurement via the TSM in "double capacitor" configuration consists of two steps, as shown in Figure 4 [18]:

1. during conditioning, the DC voltage is applied to the middle electrode and the current amplifier is short-circuited. Thus, the two cables constitute two identical capacitances in parallel with respect to the HVDC source (Figure 4.a);

2. during the measurement, the HVDC generator is disconnected for preventing the transport of induced charges at the electrodes via the HV source rather than via the current amplifier (Figure 4.b). The TS is applied to the cable under measurement (in contact with the thermal diffuser) and the TS current is measured while the current amplifier is connected to 
the compensation cable. This time, the two cables are in series with each other and with the current amplifier, and the shortcircuit condition required by the TSM is matched.

\section{RECOMMENDED PROTOCOL: THE FIRST PROPOSAL AND ITS LIMITS}

The protocol for space charge measurements in full-size HVDC extruded cables was proposed for the first time in 2011 [21], and illustrated in more detail in 2012 [22]. Since its first proposal, the space charge measurement protocol is intended to measure the spatial distributions of space charge and electric field in the insulation of full-size cables during PQ and TT load cycle tests [19].

As emphasized above, there is no universal agreement about acceptable limits for either measured space charge or electric field [20]. However, space charge and field profiles that do not attain a steady state value at the end of the poling time, show that the insulation has a tendency to undergo Poissonian field enhancements under the applied DC voltage, and is therefore considered to be unsatisfactory for service conditions. This is notwithstanding the observations that the absolute value of the amount of space charge appears to be strongly affected by measuring technique, poling procedure, cable design and even calibration procedure [20]. Despite these uncertainties, a stable field profile attained by the end of the poling time, i.e. one that does not exhibit any significant field magnification compared to the design field, is a sign of a stable charge distribution or a sign of a negligible amount of space charge, and is therefore an indicator of satisfactory performance. This holds also when comparing the performances of the as-manufactured cable with those of the aged cable after the prequalification or type test: a comparison between the field profiles measured before the test period and those measured during or after the test period enables field enhancements to be observed that are potentially harmful for cable insulation in service. An alternative view is that a change in space charge accumulation could be a sign of an ageing process during the test. This relates to the question whether space charge is the result or the initiator of an ageing process [38].

For this reason, since the very first proposal [21, 22], the protocol has prescribed that each measurement should be provided in terms of space charge density and electric field profile in the insulation wall, and that the stabilization of field profiles should be sought based on the maximum percent variation between the electric field profiles at two subsequent measurement times, $t_{l}$ and $t_{m}$. Defining such maximum percent variation as $\Delta E_{\max }\left(t_{l}-t_{m}\right)$, it can be evaluated as follows:

$\Delta E_{\max }\left(t_{l}-t_{m}\right)=\max \left\{100 \times\left|\frac{E\left(t_{l}, r_{k}\right)-E\left(t_{m}, r_{k}\right)}{E\left(t_{l}, r_{k}\right)}\right|\right\}, k=1, . ., N$

where $E\left(t, r_{k}\right)$ is the $k^{\text {th }}$ point of the electric field profile at measurement time $t$ and position $r_{k} \in\left[r_{i}, r_{o}\right]$ within the insulation; $k=1, . ., N$, being $N$ the number of points in the field profile, $r_{i}$ inner insulation radius, $r_{o}$ outer insulation radius.

As far as poling time, depolarization time, heating and cooling of specimens, since the first proposal the procedure has accounted for the previous experimental practices of space charge measurements in the thick insulation of coaxial cables, mentioned at Section 2 [2, 10, 11, 14-18]. In particular:

- a 6-hour poling time under both positive and negative voltage has been selected, since either 3 hours [14-16], or 10,000 seconds, or a few hours appear in many reference literature papers as either common poling times, or - more importantly - as times for the stabilization of charge and field profiles in HVDC insulation that is considered satisfactory [11-18, 29-33]: for instance, see Figure 1 (after [16]);

- a 2-hour depolarization time has been selected after poling with both positive and negative voltage, since in the literature depolarization times range from 1 to 3 hours as the typical times for the depletion of shallower charge traps;

- in the literature, stable charge and field profiles do show a negligible variation with time, say, within a few percent. Moreover, as an upper limit, in [39] it is found that typically in well-designed HVDC insulation the field enhancement due to space charge formation compared to average field does not exceed $20 \%$ under isothermal conditions, and 20\%-35\% under temperature gradients (although a far lower effect of temperature gradient is shown e.g. in $[13,35])$. Thus $10 \%$ is chosen as a compromise as the criterion for the stabilization of field profiles.

The first proposal after [21, 22] has been extensively reviewed and improved by the IEEE DEIS "HVDC Cable Systems" Technical Committee in the present position paper to account for some practical suggestions coming from the measurements carried out during the qualification tests relevant to ongoing HVDC projects of major significance mentioned in Section 1. The main suggestions, by either the manufacturers or the measuring teams, are as follows:

- while the first proposal only prescribed space charge measurements after the completion of the PQ or TT load cycles, a preliminary measurement should be carried out on a virgin cable sample before starting the PQ or TT load cycles for calibration purposes, in order to derive reference field profiles that will be compared with those attained at the end of PQ or TT load cycles. This can be done provided that cross-linking by-products for virgin extruded insulation do not dominate the space charge profiles; this should be the case as a long degassing time is used for HVDC extruded cables (far longer than for HVAC extruded cables);

- the measurement period during voltage-on should be (much) longer, in order to allow the stabilization of field profiles in the thick insulation of full-size cables whatever the design of the tested cables. In this respect, the reference can be the 10 hours stabilization that reflect the 
electrical dynamics present within extruded insulations for HVDC, as highlighted in [19]: "the temperature conditions as defined in $\$ 1.5 .5$ are achieved for at least 10 hours and the test object shall have been subjected to $U_{0} \ldots$ for at least 10 hours. These conditions have been selected to reflect the electrical dynamics present within extruded insulations used for HVDC" (after [19], § 1.5.6.2);

- the overall poling times and depolarization times, as well as the times for checking the stabilization of field profiles by computing their maximum absolute variation (referred to as "stabilization check-times" from now on), should be the same for both the PEA and the TSM technique;

- grounding times and procedures in passing from volt-on to volt-off ${ }^{3}$ measurements should be better defined;

- the conductor temperature and the temperature gradient across the insulation wall should be carefully defined;

- the duration of the space charge measurements should be based on the typical "working-day", thus they should be completed within no more than $\approx 8-12$ hours;

- the stabilization of field profiles should be checked on the basis of the maximum percent field variation, but the location of maximum absolute field values should be recorded, too. Indeed, a same percent variation can be far more harmful if it takes place at a location of high field;

- real time processing of space charge profiles in order to derive electric field profiles is not possible at present. However, it could be in the near future. Hence the possibility to stop the measurements, especially during volt-on, whenever the stabilization of field profiles can be assessed in real time, should be kept as an option.

Based on these suggestions, the protocol has been significantly refined and - hopefully - improved compared to the very first proposal. In particular:

- the measurements are prescribed not only at the end, but also at the beginning of PQ and TT load cycles (a further suggestion about extending the measurements throughout PQ and TT load cycles is very interesting, but it would complicate the protocol excessively at this stage, thus is still under consideration by the TC, see Section 5.B);

- the poling time under either polarity has been prolonged from 6 to 9 hours, thereby approaching the 10 hours of full stabilization of "the electrical dynamics present within extruded insulations used for HVDC" [19] and in the meanwhile matching the "working-day" basis;

- grounding times and procedures in passing from volt-on to volt-off measurements are defined more precisely, as well as conductor temperature and temperature gradient across cable insulation throughout the test;

- the maximum values of electric field in the vicinity of the inner and of the outer semicon have been added as other important quantities of the field profiles to be monitored.

\footnotetext{
${ }^{3}$ With "volt-off" it is meant - as in some reference papers about PEA measurements, see e.g. [31] - that the cable is in short-circuit conditions, thus there is no voltage applied across the cable insulation and so no charge on the electrodes except that induced by the space charge in the insulation bulk. The phrase "volt-off" is preferred here to the perhaps-clearer phrase "short-circuit" for the sake of brevity and as opposed to "volt-on", that denotes the opposite situation when a poling voltage is applied across the cable insulation.
}

This point deserves more attention. Indeed, the Poissonian field in HVDC cables often exhibits two relative maxima (see Figure 1): a first one, $E\left(t, r_{\text {inner }}\right)$, at a location $r_{\text {inner }}$ closer to the inner semicon; a second one, $E\left(t, r_{\text {outer }}\right)$, at a location $r_{\text {outer }}$ closer to the outer semicon. In the new version of the protocol, $E\left(t, r_{\text {inner }}\right)$ and $E\left(t, r_{\text {outer }}\right)$ shall be recorded at the stabilization check-times; moreover, the absolute percent variation of $E\left(t, r_{\text {inner }}\right)$ and $E\left(t, r_{\text {outer }}\right)$ between two consecutive stabilization check-times, $t_{l}$ and $t_{m}$, shall be evaluated as well, namely

$$
\begin{aligned}
& \Delta E_{\text {inner }}\left(t_{l}-t_{m}\right)=100 \times\left|\frac{E\left(t_{l}, r_{\text {inner }}\right)-E\left(t_{m}, r_{\text {inner }}\right)}{E\left(t_{l}, r_{\text {inner }}\right)}\right| \\
& \Delta E_{\text {outer }}\left(t_{l}-t_{m}\right)=100 \times\left|\frac{E\left(t_{l}, r_{\text {outer }}\right)-E\left(t_{m}, r_{\text {outer }}\right)}{E\left(t_{l}, r_{\text {outer }}\right)}\right|
\end{aligned}
$$

Although in [31] a start was made with performing space charge measurements on models of cable joints, space charge measurement set-ups for full-scale accessories are yet to come. Therefore, the protocol focuses on cables only for the moment.

\section{RECOMMENDED PROTOCOL: THE NEW PROPOSAL}

In recognition that the PEA technique is more widely reported in the literature for measurements of full-size HVDC extruded cables [11, 14-17], this method is recommended in the protocol; however, the TSM can also be used (see Sections 1 and 2). For this reason, the space charge measurement procedures to be followed when using the PEA and the TSM technique are illustrated in the sequence below. Obviously, if the values of the electric field profiles in the insulation walls of two different cables have to be compared directly (e.g. an already-qualified cable design vs. an innovative cable design not yet qualified), the same space charge measurement method shall be employed for both cables, i.e. either the PEA or the TSM. However, this aspect is beyond the main scope of the protocol. The focus in the protocol is on the variation of the field profiles in order to check for the attainment of stable field profiles as an indication of satisfactory and reliable performances. From this respect, both methods are expected to provide concordant indications as to the attainment of stable field profiles.

\subsection{PREQUALIFICATION TESTS}

\subsubsection{PEA METHOD DURING PQ TEST}

The recommended protocol - as detailed below in points 1 to 14 - shall be followed for performing space charge measurements via the PEA method at the following times during the long-term prequalification test after [19]:

a) before starting the PQ load cycles. This first space charge measurement will serve as a reference for calibration purposes and shall be performed on a virgin 
cable sample identical to that employed in the PQ loop as defined in [19];

b) after the completion of the PQ load cycles.

Note that, as far as the two above items a) and b) are concerned, two possible testing schemes shall be considered for practical reasons:

1) In the first scheme, space charge measurements shall all be made on a parallel loop, subjected to the same PQ load cycles as the main loop, to be measured before and after the PQ load cycles. Since the measurements require that the outer shielding layer is removed - and this may change the de-gassing rate and the effect of humidity on the measured cable compared to the rest of the loop - this can be done provided that the humidity in the lab is carefully controlled and that cross-linking by products for virgin extruded insulation do not dominate the space charge profiles, as pointed out in Section 3.

2) As an alternative to the first scheme i) a space charge measurement before PQ load cycles is performed at a location of the main loop close to one termination; ii) after this measurement such cable section is cut, and a joint is installed; iii) PQ load cycles are performed; iv) the measurements after PQ load cycles ensue on the main loop, whose overall initial length should be sufficient.

In both cases above, an adequate section of the outer semicon of the cable shall be exposed where the PEA measurement cell is to be made. All the required power and signal connections shall be setup. Then, the space charge measurements shall consist of the following steps.

1. The cable shall be heated, and the conductor kept for at least 24 hours at a value $T_{\text {cond,max }}$, with a temperature gradient $\Delta T$ across cable insulation - where $T_{\text {cond, } \max }$ and $\Delta T$ are the values stated by [19] for the PQ test - before the space charge measurements start. Later on, the cable conductor $T_{\text {cond,max }}$ and the temperature gradient $\Delta T$ across cable insulation shall be maintained for the whole duration of the space charge measurements. For ensuring that $T_{\text {cond,max }}$ and $\Delta T$ are the same also in the cable section with exposed semicon where the PEA test cell is located, this cable section shall be covered with proper thermal insulation coatings throughout all the measurements.

2. The rated voltage $U_{0}$ of the tested cable system (as defined in [19]) shall be applied between the conductor and the metal sheath (grounded) with positive polarity: the positive voltage poling time, $t_{P V, O N}$, will start.

3. Immediately after the attainment of the rated voltage, i.e. at $t_{P V, O N}=0^{+}$, a first volt-on measurement shall be performed, that represents the volt-on reference at the start of the positive polarity measurements.

4. A first sequence of 3 volt-on measurements shall be performed every $60 \mathrm{~min}$ ( 1 hour), until a time equal to 180 min ( 3 hours) has passed since $t_{P V, O N}=0^{+}$. Thus, the sequence of measurement times is $t_{P V, O N}(1)=60 \mathrm{~min}$, $t_{P V, O N}(2)=120 \mathrm{~min}, t_{P V, O N}(3)=180 \mathrm{~min}=3 \mathrm{~h}$. The relevant space charge and electric field profiles within the insulation wall shall be recorded and plotted ${ }^{4}$.

\footnotetext{
${ }^{4}$ All recorded, calculated and plotted quantities shall appear in the test report.
}

5. The maximum absolute percent variation between the electric field profile at $t_{P V, O N}=0^{+}$and at $t_{P V, O N}(3)=3 \mathrm{~h}-$ $\Delta E_{\max }(0-3 \mathrm{~h})$ - shall be evaluated from equation (1), as follows:

$\Delta E_{\max }(0-3 h)=\max \left\{100 \times\left|\frac{E\left(0, r_{k}\right)-E\left(3 h, r_{k}\right)}{E\left(0, r_{k}\right)}\right|\right\}, k=1, . ., N(4)$

Moreover the locations and the values of the maximum field at $t_{P V, O N}(3)=3 \mathrm{~h}$ in the vicinity of the inner electrode, $r_{\text {inner }}$, and of the outer electrode, $r_{\text {outer }}$, will be recorded, and the relevant variations with respect to the electric field values at $t_{P V, O N}=0^{+}-\Delta E_{\text {inner }}(0-3 \mathrm{~h})$ and $\Delta E_{\text {outer }}(0-3 \mathrm{~h})$, respectively - shall be evaluated for engineering purposes from equations (2) and (3), as follows:

$$
\begin{aligned}
& \Delta E_{\text {inner }}(0-3 h)=100 \times\left|\frac{E\left(0, r_{\text {inner }}\right)-E\left(3 h, r_{\text {inner }}\right)}{E\left(0, r_{\text {inner }}\right)}\right| \\
& \Delta E_{\text {outer }}(0-3 h)=100 \times\left|\frac{E\left(0, r_{\text {outer }}\right)-E\left(3 h, r_{\text {outer }}\right)}{E\left(0, r_{\text {outer }}\right)}\right|
\end{aligned}
$$

Stabilization is assumed as having occurred at $t_{P V, O N}(3)=3$ $\mathrm{h}$ if: $\Delta E_{\max }(0-3 \mathrm{~h}) \leq 10 \%$.

6. In case that the achievement of stabilization can be proved in real time, go to step 10. Otherwise, a second sequence of 3 volt-on measurements shall be performed every 60 min (1 hour), until an overall time equal to 360 min (6 hours) has passed since $t_{P V, O N}=0^{+}$. Thus, the sequence of further measurement times is $t_{P V, O N}(4)=240$ $\min , t_{P V, O N}(5)=300 \mathrm{~min}, t_{P V, O N}(6)=360 \mathrm{~min}=6 \mathrm{~h}$. The relevant space charge and electric field profiles within the insulation wall shall be recorded and plotted.

7. The maximum absolute percent variation between the electric field profile at $t_{P V, O N}(3)=3 \mathrm{~h}$ and $t_{P V, O N}(6)=6 \mathrm{~h}-$ $\Delta E_{\max }(3 \mathrm{~h}-6 \mathrm{~h})$ - shall be evaluated from equation (1). Moreover the locations and the values of the maximum field at $t_{P V, O N}(6)=6 \mathrm{~h}$ in the vicinity of the inner electrode, $r_{\text {inner }}$, and of the outer electrode, $r_{\text {outer }}$, will be recorded, and the relevant variations with respect to the electric field values at $t_{P V, O N}(3)=3 \mathrm{~h}-\Delta E_{\text {inner }}(3 \mathrm{~h}-6 \mathrm{~h})$ and $\Delta E_{\text {outer }}(3 \mathrm{~h}-6 \mathrm{~h})$, respectively - shall be evaluated for engineering purposes from equation (2) and (3). Detailed expressions are omitted for brevity. Stabilization is assumed as having occurred at $t_{P V, O N}(6)=6 \mathrm{~h}$ if: $\Delta E_{\max }(3 \mathrm{~h}$ $6 \mathrm{~h}) \leq 10 \%$.

8. In case that the achievement of stabilization can be proved in real time, go to step 10. Otherwise, a final sequence of 3 volt-on measurements shall be performed every $60 \mathrm{~min}$ (1 hour), until an overall time equal to 540 min (9 hours) has passed since $t_{P V, O N}=0^{+}$. Thus, the sequence of further measurement times is $t_{P V, O N}(7)=420$ $\min , t_{P V, O N}(8)=480 \mathrm{~min}, t_{P V, O N}(9)=540 \mathrm{~min}=9 \mathrm{~h}$. The relevant space charge and electric field profiles within the insulation wall thickness shall be recorded and plotted. 
9. The maximum percent variation between the electric field profile at $t_{P V, O N}(9)=540 \mathrm{~min}$ and at $t_{P V, O N}(6)=360 \mathrm{~min}-$ $\Delta E_{\max }(6 \mathrm{~h}-9 \mathrm{~h})$ - shall be evaluated from equation (1). Moreover the locations and the values of the maximum field at $t_{P V, O N}(9)=9 \mathrm{~h}$ in the vicinity of the inner electrode, $r_{\text {inner }}$, and of the outer electrode, $r_{\text {outer }}$, will be recorded, and the relevant variations with respect to the electric field values at $t_{P V, O N}(6)=6 \mathrm{~h}-\Delta E_{\text {inner }}(6 \mathrm{~h}-9 \mathrm{~h})$ and $\Delta E_{\text {outer }}(6 \mathrm{~h}-9 \mathrm{~h})$, respectively - shall be evaluated for engineering purposes from equations (2) and (3). Detailed expressions are omitted for brevity. Stabilization is assumed as having occurred at $t_{P V, O N}(9)=9 \mathrm{~h}$ if: $\Delta E_{\max }(6 \mathrm{~h}-$ $9 h) \leq 10 \%$.

10. The applied voltage shall be reduced and then conductor shall be short-circuited and grounded: voltage reduction and grounding shall be performed within a time no longer than $5 \mathrm{~min}$. Both the cable conductor and the HV electrode of the HVDC source employed in the test will be solidly and effectively grounded, without interposed resistances, and kept grounded throughout the volt-off measurements until the volt-on measurements with negative polarity starts (see point 14 below).

11. Immediately after the cable grounding, the positive voltage depolarization time, $t_{P V, O F F}$, will start and a first volt-off measurement shall be performed at $t_{P V, O F F}=0^{+}$, i.e. the volt-off reference measurement at the start of depolarization after poling the cable with positive polarity.

12. A sequence of three volt-off measurements shall be performed every $60 \mathrm{~min}$, until a time of $180 \mathrm{~min}$ has passed. Thus, the sequence of measurement times is $t_{P V, O F F}(1)=60 \mathrm{~min}, t_{P V, O F F}(2)=120 \mathrm{~min}, t_{P V, O F F}(3)=180$ $\min =3 \mathrm{~h}$. The relevant space charge and electric field profiles within the insulation wall shall be recorded and plotted.

13. The cable shall be left grounded for at least $24 \mathrm{~h}$.

14. After the completion of step 13, the rated voltage $U_{0}$ shall be applied between conductor and metal sheath (grounded) with negative polarity; the negative voltage poling time, $t_{N V, O N}$, will start and thereafter the whole procedure from step 2 to step 12 shall be repeated.

\subsubsection{THERMAL STEP METHOD (TSM) DURING PQ TEST}

Two cable lengths of $10 \mathrm{~m}$ at least shall be arranged in such a way that the electric field and space charges can be measured using the TSM-double capacitor, outer cooling procedure [18]. The double capacitor configuration involves using two identical cables for the test set-up: the first is subjected to space charge measurements, the second serves for determining the thermal step current associated with space charge in the first cable subjected to measurement by compensating the polarization and conduction currents flowing across the insulation under high DC field (see Section 2.2) [18].

The recommended protocol - as detailed here below at points from 1 to 14 - should be followed for performing space charge measurements using the TSM technique at the following times during the long-term prequalification test after [19]:

a) before starting the long-term PQ test. This first space charge measurement will serve as a reference for calibration purposes and shall be performed on two virgin cable samples identical to that employed in the PQ loop as defined in [19];

b) after the completion of the long-term PQ test.

In both cases above, an adequate section of the outer semicon of the measured cable shall be exposed where the TSM set-up is to be used. All the required power and signal connections shall be setup. Then, the space charge measurements shall consist of the following steps.

1. The cable shall be heated, and the conductor kept for at least 24 hours at a value $T_{\text {cond,max }}$, with a temperature gradient $\Delta T$ across cable insulation - where $T_{\text {cond,max }}$ and $\Delta T$ are the values stated by [19] for the PQ test - before the space charge measurements start. Later on, the cable conductor $T_{\text {cond,max }}$ and the temperature gradient $\Delta T$ across cable insulation shall be maintained for the whole duration of the space charge measurements. For ensuring that $T_{\text {cond,max }}$ and $\Delta T$ are the same also in the cable section with exposed semicon where the thermal diffuser is located, this cable section shall be covered with proper thermal insulation coatings throughout all the measurements.

2. The rated voltage $U_{0}$ of the tested cable system (as defined in [19]) shall be applied between the conductor and the metal sheath (grounded) with positive polarity: the positive voltage poling time, $t_{P V, O N}$, starts.

3. Immediately after the attainment of the rated voltage, i.e. at $t_{P V, O N}=0^{+}$, a first volt-on measurement shall be performed, that represents the volt-on reference at the start of the positive polarity measurements.

4. A first sequence of 2 volt-on measurements shall be performed every $90 \mathrm{~min}$ (11/2 hour), until a time equal to $180 \mathrm{~min}$ (3 hours) has passed. Thus, the sequence of measurement times is $t_{P V, O N}(1)=90 \mathrm{~min}, t_{P V, O N}(2)=180$ $\min =3 \mathrm{~h}$. The relevant space charge and electric field profiles within the insulation wall shall be recorded and plotted.

5. The maximum absolute percent variation between the electric field profile at $t_{P V, O N}=0^{+}$and at $t_{P V, O N}(2)=3 \mathrm{~h}-$ $\Delta E_{\max }(0-3 \mathrm{~h})$ - shall be evaluated according to equation (4). Moreover the locations and the values of the maximum field at $t_{P V, O N}(2)=3 \mathrm{~h}$ in the vicinity of the inner electrode, $r_{\text {inner }}$, and of the outer electrode, $r_{\text {outer }}$, will be recorded, and the relevant variations with respect to the electric field values at $t_{P V, O N}=0^{+}-\Delta E_{\text {inner }}(0-3 \mathrm{~h})$ and $\Delta E_{\text {outer }}(0-3 \mathrm{~h})$, respectively - shall be evaluated for engineering purposes according to equations (5) and (6). Stabilization is assumed as having occurred at $t_{P V, O N}(2)=3$ $\mathrm{h}$ if: $\Delta E_{\max }(0-3 \mathrm{~h}) \leq 10 \%$.

6. In case that the achievement of stabilization can be proved in real time, go to step 10. Otherwise, a second sequence of 2 volt-on measurements shall be performed every $90 \mathrm{~min}\left(1 \frac{1}{2}\right.$ hour), until an overall time equal to 360 
min (6 hours) has passed since $t_{P V, O N}=0^{+}$. Thus, the sequence of further measurement times is $t_{P V, O N}(3)=270$ $\min , t_{P V, O N}(4)=360 \min =4 \mathrm{~h}$. The relevant space charge and electric field profiles within the insulation wall shall be recorded and plotted.

7. The maximum absolute percent variation between the electric field profile at $t_{P V, O N}(2)=3 \mathrm{~h}$ and $t_{P V, O N}(4)=6 \mathrm{~h}-$ $\Delta E_{\max }(3 \mathrm{~h}-6 \mathrm{~h})$ - shall be evaluated from equation (1). Moreover the locations and the values of the maximum field at $t_{P V, O N}(4)=6 \mathrm{~h}$ in the vicinity of the inner electrode, $r_{\text {inner }}$, and of the outer electrode, $r_{\text {outer }}$, will be recorded, and the relevant variations with respect to the electric field values at $t_{P V, O N}(2)=3 \mathrm{~h}-\Delta E_{\text {inner }}(3 \mathrm{~h}-6 \mathrm{~h})$ and $\Delta E_{\text {outer }}(3 \mathrm{~h}-6 \mathrm{~h})$, respectively - shall be evaluated for engineering purposes from equations (2) and (3). Detailed expressions are omitted for brevity. Stabilization is assumed as having occurred at $t_{P V, O N}(4)=6 \mathrm{~h}$ if: $\Delta E_{\max }(3 \mathrm{~h}-$ $6 \mathrm{~h}) \leq 10 \%$.

8. In case that the achievement of stabilization can be proved in real time, go to step 10. Otherwise, a final sequence of 2 volt-on measurements shall be performed every $90 \mathrm{~min}\left(1 \frac{1}{2}\right.$ hour), until an overall time equal to 540 min (9 hours) has passed since $t_{P V, O N}=0^{+}$. Thus, the sequence of further measurement times is $t_{P V, O N}(5)=450$ $\min , t_{P V, O N}(6)=540 \min =9 \mathrm{~h}$. The relevant space charge and electric field profiles within the insulation wall shall be recorded and plotted.

9. The maximum percent variation between the electric field profile at $t_{P V, O N}(6)=540 \mathrm{~min}$ and at $t_{P V, O N}(4)=360 \mathrm{~min}-$ $\Delta E_{\max }(6 \mathrm{~h}-9 \mathrm{~h})$ - shall be evaluated from equation (1). Moreover the locations and the values of the maximum field at $t_{P V, O N}(6)=9 \mathrm{~h}$ in the vicinity of the inner electrode, $r_{\text {inner }}$, and of the outer electrode, $r_{\text {outer }}$, shall be recorded, and the relevant variations with respect to the electric field values at $t_{P V, O N}(4)=6 \mathrm{~h}-\Delta E_{\text {inner }}(6 \mathrm{~h}-9 \mathrm{~h})$ and $\Delta E_{\text {outer }}(6 \mathrm{~h}-9 \mathrm{~h})$, respectively - shall be evaluated for engineering purposes from equations (2) and (3). Detailed expressions are omitted for brevity. Stabilization is assumed as having occurred at $t_{P V, O N}(6)=9 \mathrm{~h}$ if: $\Delta E_{\max }(6 \mathrm{~h}-$ $9 \mathrm{~h}) \leq 10 \%{ }^{5}$.

10. The applied voltage shall be reduced and then conductor shall be short-circuited and grounded: voltage reduction and grounding shall be performed within a time no longer than $5 \mathrm{~min}$. Both the cable conductor and the HV electrode of the HVDC source employed in the test will be solidly and effectively grounded, without interposed resistances, and kept grounded throughout the volt-off measurements until the volt-on measurements with negative polarity starts (see point 14 below).

11. Immediately after the cable grounding, the positive voltage depolarization time, $t_{P V, O F F}$, will start and a first volt-off measurement shall be performed at $t_{P V, O F F}=0^{+}$, i.e. the volt-off reference measurement at the start of depolarization after poling the cable with positive polarity.

\footnotetext{
${ }^{5}$ In this way the whole poling time $(9 \mathrm{~h}$ at worst) and the 3 stabilization check-times ( $3 \mathrm{~h}, 6 \mathrm{~h}, 9 \mathrm{~h})$ are the same for PEA and TSM
}

12. A sequence of two volt-off measurements shall be performed every $90 \mathrm{~min}$, until a time of $180 \mathrm{~min}=3 \mathrm{~h}$ has passed. Thus, the sequence of measurement times is $t_{P V, O F F}(1)=90 \mathrm{~min}, t_{P V, O F F}(2)=180 \mathrm{~min}^{6}$. The relevant space charge profiles and electric field profiles within the insulation wall thickness shall be recorded and plotted.

13. The cable shall be left grounded for at least $24 \mathrm{~h}$.

14. After the completion of step 13, the rated voltage shall be applied between conductor and screen (grounded) with negative polarity; the negative voltage poling time, $t_{N V, O N}$, starts and thereafter the whole procedure from step 2 to step 12 shall be repeated.

\subsection{TYPE TESTS}

\subsubsection{PEA METHOD DURING TYPE TEST}

The recommended protocol - as detailed below - shall be followed for performing space charge measurements using the PEA method at the following times during the type test load cycles after [19]:

a) before starting the TT load cycles. This first space charge measurement will be a reference for calibration purposes and shall be performed on a virgin cable sample identical to that employed in the TT load cycle loop as defined in [19];

b) after the completion of the TT load cycles.

Note that, as far as the two above items a) and b) are concerned, two possible testing schemes shall be considered for practical reasons, as in the case of the PQ test (see Section 4.1.A and the relevant comments):

1) in a first scheme, space charge measurements shall all be made on a parallel loop, subjected to the same TT load cycles as the main loop, to be measured before and after the TT load cycles;

2) as an alternative to the first scheme i) space charge measurement before TT load cycles is performed at a location of the main loop close to one termination; ii) after this measurement such cable section is cut, and a joint is installed; iii) TT load cycles are performed; iv) the measurements after TT load cycles ensue on the main loop, whose overall initial length should be sufficient.

The recommended protocol for PEA measurements in fullsize HVDC extruded cables during the TT load cycles after [19] consists of the same steps listed in Section 4.1.A for the measurements during PQ tests.

\subsubsection{THERMAL STEP METHOD (TSM) DURING TYPE TEST}

Two cable lengths of $10 \mathrm{~m}$ at least shall be arranged in such a way that the electric field and space charges can be measured using the TSM-double capacitor, outer cooling procedure [18] (see Section 4.1.B).

The recommended protocol - as detailed here below - shall be followed for performing space charge measurements via

\footnotetext{
${ }^{6}$ In this way the whole volt-off time ( $3 \mathrm{~h}$ ) is the same for PEA and TSM, and two common time instant exist: $0,3 \mathrm{~h}$. The overall measurement time is $12 \mathrm{~h}$ at worst, feasible in a single day.
} 
the TSM technique at the following times during the type test load cycles after [19]:

a) before starting the TT load cycles. This first space charge measurement will be a reference for calibration purposes and shall be performed on two virgin cable samples identical to that employed in the TT loop as defined in [19]. The cable section that undergoes this preliminary space charge measurement will be subsequently subjected to the same temperature and voltage cycles as the whole TT loop, and will eventually undergo the measurements detailed at next item b);

b) after the completion of the TT load cycles. This space charge measurement will be performed on the same cable section as at previous item a).

The recommended protocol for TSM measurements in fullsize HVDC extruded cables during the TT load cycles after [19] consists of the same steps listed in Section 4.1.B for the same measurements during PQ tests.

\section{DISCUSSION OF THE PROTOCOL}

\subsection{DIFFICULTIES IN THE MEASUREMENTS ON FULL-SIZE CABLES}

Whatever the chosen technique, several problems may be encountered in the measurement of space charge on large size HVDC cables. First of all, large HV terminations have to be mounted, that require an adequate cable length. According to the practical experience, a full-size cable length of at least $10 \mathrm{~m}$ should be selected to connect the terminations in a proper way for performing the space charge measurements.

Moreover, the grounding system of the measurement set-up requires particular care in order to avoid spurious signals being gathered, which lead to excessive electrical noise. The inductance of the grounding system has also to be controlled, in order to avoid undesired oscillations superimposed on the space charge signal.

Focusing on the PEA technique, more problems may arise from the low signal-to-noise ratio combined with the frequency response of transducer and amplifier, since - as pointed out in [40] - "in some cases they may distort the acoustic wave. As a result, part of the output signal may be wrongly interpreted as space charge... Distortion takes place if the frequency response of the amplifier is not flat, if the amplifier's high cut-off frequency is too low, or if the combination of transducer and amplifier act as a high-pass filter. The first two problems are easily solved by using a high quality amplifier with good and sufficient frequency characteristics... The third problem can be solved mathematically or by using an amplifier with an higher input impedance, for instance $50 \mathrm{k} \Omega$. The disadvantage of such amplifiers is that their noise level is much higher". The laboratories of manufacturers are typically electrically noisy environments leading to low signal-to-noise ratios. Low-noise amplifiers are therefore desirable for space charge measurements on full-size cables tested in such labs but often these have a low input impedance (typically $50 \Omega$ ).
Another problem in PEA measurement on full-size cables is the considerable thickness of their insulation that can be as high as $\sim 20 \mathrm{~mm}$ or more. As a consequence, pressure waves coming from the vicinity of the inner electrode (i.e. the conductor) are highly attenuated and distorted. This leads to calibration problems, which can, however, be overcome, as described in [14]. The selection of the height and width of the voltage pulses should also account for this noting that:

- for the purposes of this protocol, there is no need for a very high spatial resolution as the full-size cables have a thick insulation, thus the pulses do not need to be very narrow;

- the height of the pulses should not be excessive in order to avoid problems with the measuring instrumentations and generation of space charge associated with the pulses themselves [20].

Furthermore, due to the considerable length of the measured cable, undesired reflections of the signal at the far end could arise notwithstanding the experimental set-up illustrated in Figure 2. These reflections, as well as the high frequency noise, should be analysed and removed via a careful treatment of the signal, which requires considerable skills and experience at the post-processing stage.

Turning to the TSM, it must be pointed out that this method is more cumbersome to use both as to the measurement set-up (two identical cable samples needed) and as to the measurement procedure (it requires disconnecting the HVDC source and circulating a cold liquid during the measurements, see Section 2.2). Thus the frequency of the measurements should be much lower when using the TSM than when using the PEA; this also explains the longer time interval between two consecutive stabilization check-times prescribed by the protocol in the case the TSM is used (see Sections 4.1.B, 4.2.B).

\subsubsection{MEASUREMENTS WITHIN QUALIFICATION LOAD CYCLES}

As suggested by some manufacturers, it would be very interesting to extend the space charge measurements and the relevant protocol to the period within PQ and TT load cycles, since more information about cable behaviour would be acquired in this way. However, some difficulties arise.

First of all, the prescribed cable temperature and voltage should be modified in order to account for the temperature gradient and the applied voltage that are actually present at the various times within load cycles. For instance, taking PQ load cycles as a reference, the applied DC voltage is higher than the rated voltage throughout the PQ load cycles; the conductor is kept at a value no lower than $T_{\text {cond, } \max }$ - with a temperature gradient $\Delta T$ across cable insulation - only during the so-called High Load (HL) period of the cycles, lasting for 80 cycles, while during the other 280 cycles the conductor temperature and the gradient across the insulation are mostly lower than $T_{\text {cond,max }}$ and $\Delta T$. Secondly, the volt-off measurements cannot be performed and the voltage polarity cannot be reversed during the load cycles, unless the measurements coincide with the end of the various load 
cycle parts (see [19], details are omitted for the sake of brevity). Finally, the experience of space charge measurement on full-size cables within qualification load cycles is scarce. Furthermore, in the case that the TSM is used, strictly speaking the measurements could not be performed during qualification load cycles, since the HVDC source cannot be disconnected from the sample without interrupting the qualification, which is not acceptable.

For these and other reasons - not treated here for brevity -the IEEE DEIS Technical Committee "HVDC Cable Systems" has decided that extending space-charge measurements throughout PQ and TT load cycles would complicate too much the protocol at this stage, although such extension is currently under consideration by the committee.

\subsubsection{VALIDATION OF THE PROTOCOL}

The protocol in its former version following the first proposal $[21,22]$ has already been applied to the space charge measurements performed in some laboratories worldwide on full-size cable loops during qualification tests for HVDC extruded cable link projects of major significance. The measurements were all performed after PQ and TT load cycles. The protocol has proven to be successful. Indeed:

- the manufacturers have accepted the protocol, taking it as a chance for a deeper assessment of the performances of their cables and even suggesting in some cases a longer poling time;

- the teams that performed the measurements have judged the protocol positively, and have contributed to its improvement by providing useful suggestions (see Section $3)$;

- the prescriptions of the protocol have been shown to be feasible in all respects, in particular as to the time schedule, the procedures for switching on and off the voltage, for processing the results, and so on;

- although details of the measurement results cannot be disclosed, being classified, the results proved that all cables that passed the qualification load cycles successfully did show a stabilization of the field profiles after 3 to 6 hours of poling. Thus the selected time basis for performing the volton measurements is not only supported by former experience, but also confirmed by these latter measurements of great significance, being relevant to very different cable designs.

\section{CONCLUSIONS}

The proposed protocol according to the first proposal has been applied successfully to the space charge measurements using either the PEA or the TSM technique performed in some laboratories worldwide on full-size HVDC extruded cable loops at the end of qualification (PQ and TT) load cycles for HVDC extruded cable link projects of major significance. The protocol has proven to be successful. Indeed, the teams that performed the measurements favorably accepted the protocol, and cooperated to its improvement. Moreover, the manufacturers took it as a chance for deepening the knowledge of the performances of their cables. In addition the prescriptions of the protocol have been shown to be feasible in all respects and although details of the measurement results are classified all cables that passed successfully the qualification load cycles did show a stabilization of the field profiles within 3 to 6 hours of poling.

Since the new version of the protocol has been improved in many respects, it can be expected to work properly in the foregoing measurement campaigns. The experience that will be gained in such campaigns will help to better focus on some aspects, and in particular to propose an extension of the space charge measurements also to times within the qualification load cycles.

Aspects to be focused on in the next future include more frequent measurements of space charge during testing and online monitoring of cables. This will be the next goal of the IEEE DEIS Technical Committee "HVDC Cable Systems.

\section{REFERENCES}

[1] G. Mazzanti and M. Marzinotto, Extruded Cables for High Voltage Direct Current Transmission: Advances in Research and Development, Power Engineering Series - Wiley-IEEE Press, ISBN 978-1-118-096666, 2013.

[2] R. Liu, "Long-distance DC electrical power transmission", IEEE Electr. Insul. Mag., Vol. 29, No. 5, pp. 37-46, 2013.

[3] R. Rendina, M. R. Guarniere, R. Niccolai, G. Pazienza, A. Gualano, S. Malgarotti, A. Danelli, B. Jansson, F. Alvarez, W. Lovison, A. Persico, A. Orini, F. Bocchi, S. Aleo, and G. Curtotti, "The realization and commissioning of the $\pm 500 \mathrm{kV} 1000 \mathrm{MW}$ HVDC link Sardinia island Italian peninsula (SAPEI)", CIGRE paper B1-101, 2012, pp. 1-11.

[4] Y. Ohki, "Development of XLPE-Insulated cable for high-voltage DC submarine transmission line (2)", IEEE Electr. Insul. Mag., Vol. 29, No. 5, pp. 85-87, 2013.

[5] B. Jacobson, Y. Jiang-Hafner, P. Rey, G. Asplund, M. Jeroense, A. Gustafsson, and M. Bergkvist, "HVDC with voltage source converters and extruded cables for up to $\pm 300 \mathrm{kV}$ and $1000 \mathrm{MW}$ ", CIGRE paper B4-105, 2006, pp. 1-8.

[6] R.N. Hampton, "Some of the considerations for materials operating under high-voltage, direct- current stresses", IEEE Electr. Insul. Mag., Vol. 24, No. 1, pp. 5-13, 2008.

[7] M. Jeroense, A. Gustafsson, and M. Bergkvist, "HVDC Light cable system extended to $320 \mathrm{kV}$ ", CIGRE paper B1-304, 2008, pp. 1-9.

[8] P. Lundberg, M. Callavik, M. Bahrman, and P. Sandeberg, "Platforms for change: High-voltage DC converters and cable technologies for offshore renewable integration and DC grid expansions", IEEE Power Energy Mag., pp. 30-38, 2012.

[9] Y. Ohki, "Development of XLPE-Insulated cable for high-voltage DC submarine transmission line (1)", IEEE Electr. Insul. Mag., Vol. 29, No. 4, pp. 65-67, 2013.

[10] N.H. Ahmed, and N.N. Srinivas, "Review of space charge measurements in dielectrics", IEEE Trans. Dielectr. Electr. Insul., Vol. 4, No. 5, pp. 644-656, 1997.

[11] T. Takada, "Acoustic and optical methods for measuring electric charge distributions in dielectrics", IEEE Trans. Dielectr. Electr. Insul., Vol. 6 No. 5, pp. 519-547, 1999.

[12] T.L. Hanley, R.P. Burford, R.J. Fleming, and K.W. Barber, "A general review of polymeric insulation for use in HVDC cables", IEEE Electr. Insul. Mag., Vol. 19, No. 1, pp. 14-24, 2003.

[13] M. Fu, L.A. Dissado, G. Chen, and J.C. Fothergill, "Space charge formation and its modified electric field under applied voltage reversal and temperature gradient in XLPE cable", IEEE Trans. Dielectr. Electr. Insul., Vol. 15, No. 3, pp. 851-860, 2008. 
[14] T. Takeda, N. Hozumi, H. Suzuki, K. Fujii, K. Terashima, M. Hara, Y. Murata, K. Watanabe, and M. Yoshida, "Space charge behavior in full size $250 \mathrm{kV}$ DC XLPE cables", IEEE Trans. Power Delivery, Vol. 13, No. 1, pp. 28-39, 1998.

[15] K. Terashima, H. Suzuki, M. Hara, and K. Watanabe, "Research and development of $+250 \mathrm{kV}$ DC XLPE Cables", IEEE Trans. Power Delivery, Vol. 13, No. 1, pp. 7-16, 1998.

[16] T.Yamanaka, S. Maruyama, and T.Tanaka, "The development of DC $+/-500 \mathrm{kV}$ XLPE Cable in consideration of the space charge accumulation", IEEE $7^{\text {th }}$ Int'l. Conf. Properties and Applications of Dielectric Materials (ICPADM), , Nagoya, Japan, pp. 689-694, 2003.

[17] S. Maruyama, N. Ishii, M. Shimada, S. Kojima, H. Tanaka, M. Asano, T. Yamanaka, and S. Kawakami, "Development of a $500-\mathrm{kV}$ DC XLPE cable system", Furukawa Review, No. 25, pp. 47-52, 2004.

[18] J. Castellon, P. Notingher, S. Agnel, A. Toureille, F. Brame, P. Mirebeau, and J. Matallana, "Electric field and space charge measurements in thick power cable isulation", IEEE Electr. Insul. Mag., Vol. 25, No. 3, pp. 30-42, 2009.

[19] Brochure CIGRE 496, "Recommendations for testing DC extruded cable systems for power transmission at a rated voltage up to 500kV", CIGRE WG B1-32, 2012

[20] Brochure CIGRE 288, "Space charge measurement in dielectrics and insulating materials - PEA, PWP (PIPWP \& LIPP) and TSM methods", prepared by Task Force D1.12.01, 2006.

[21] M. Marzinotto, and G. Mazzanti, "A procedure for space charge measurements in full-size HVDC extruded cables", Proc. of the 2011 IEEE Conf. Electr. Insul. Dielectric Phenomena (CEIDP), Cancun (Mexico), pp. 804-807, 2011.

[22] M. Marzinotto, and G. Mazzanti, "A procedure for space charge measurements in full-size HVDC extruded cables in order to access the electric field in the insulation wall", CIGRE Session 2012, paper D1-202, pp. 1-6, Paris (France), 2012.

[23] F.N. Lim, R.J. Fleming and R.D. Naybour, "Space charge accumulation in power cable XLPE Insulation", IEEE Trans. Dielectr. Electr. Insul., Vol. 6, pp. 273-281, 1999.

[24] F.N. Lim, R.J. Fleming and R.D. Naybour, "Space charge accumulation in power cable XLPE insulation", IEEE $3^{\text {rd }}$ Int'l. Conf. Electric Charge in Solid Insulators, Tours (France), pp. 329-338, 1998.

[25] F.N. Lim and R.J Fleming, The Influence of $\mathrm{O}_{2}, \mathrm{~N}_{2}$ and $\mathrm{H}_{2} \mathrm{O}$ on space-charge accumulation in XLPE under DC Fields, Int'l. Sympos. Electr. Insulating Materials, Toyohashi, Japan, pp. 199-202, 1998.

[26] T. Takada, T. Maeno and H. Kushibe, "An electric stress-pulse technique for the measurement of charges in a plastic plate irradiation by an electron beam", IEEE. Trans. Electr. Insul., Vol. 22, No. 4, pp. 497-501, 1987.

[27] K. Fukunaga, H. Miyata, M. Sugimori, and T. Takada, "measurement of charge distribution in the insulation of cables using pulsed electroacoustic method", Trans. IEE Japan, Vol.110-A, No. 9, pp. 647-648, 1990.

[28] N. Hozumi, H. Suzuki, T. Okamoto, K. Wakanabe, and A. Watanabe, "Direct observation of time dependent space charge profiles in XLPE cable under high electric field, IEEE Trans. Dielectr. Electr. Insul., Vol. 1, No. 6, pp.1068-1076, 1994.

[29] R. Bodega, P. H. F. Morshuis, U. H. Nilsson, G. Perego, and J.J Smit, "Charging and polarization phenomena in coaxial XLPE-EPR interfaces", in Proc. IEEE ISEIM, Kitakyushu, Japan, pp. 107-110, June 2005.

[30] D. Fabiani, G.C. Montanari, C. Laurent, G. Teyssedre, P.H.F. Morshuis, R. Bodega, L.A. Dissado, A. Campus, and U.H. Nilsson, "Polymeric HVDC cable design and space charge accumulation. Part 1: insulation/semicon interface", IEEE Electr. Insul. Mag., Vol. 23, No. 6, pp. $11-19,2007$.

[31] S. Delpino, D. Fabiani, G.C. Montanari, C. Laurent, G. Teyssedre, P.H.F. Morshuis, R. Bodega, and L.A. Dissado, "Polymeric HVDC cable design and space charge accumulation. Part 2: insulation interfaces", IEEE Electr. Insul. Mag., Vol. 24, No. 1, pp. 14-24, 2008.

[32] D. Fabiani, G.C. Montanari, C. Laurent, G. Teyssedre, P.H.F. Morshuis, R. Bodega, and L.A. Dissado, "HVDC cable design and space charge accumulation. Part 3: Effect of temperature gradient", IEEE Electr. Insul. Mag., Vol. 24, No. 2, pp. 5-14, 2008.
[33] C.O. Olsson, and M. Jeroense, "Evolution of the distributions of electric field and of space charge in an extruded HVDC cable", $8^{\text {th }}$ Int'1. Conf. Insulated Power Cables (Jicable'11), Versailles, France, Paper A.2.6, 2011.

[34] H. Mori, H. Niinobe, and Y. Yagi, "Space charge measurements and characteristics of HVDC XLPE cable", Paper P05, JicableHVDC'13, Perpignan (France), 2013.

[35] W. Choo, G. Chen and S.G. Swingler, "Electric field in polymeric cable due to space charge accumulation under DC and temperature gradient", IEEE Trans. Dielectr. Electr. Insul., Vol. 18, No. 2, pp. 596-606, 2011

[36] P. Notingher, Jr., S. Agnel, and A. Toureille, "Thermal step method for space charge measurements under applied dc field", IEEE Trans. Dielectr. Electr. Insul., Vol. 8, No. 6, pp. 985-994, 2001.

[37] A. Cherifi, M. Abou Dakka, and A. Toureille, "The Validation of the Thermal Step Method", IEEE Trans. Electr. Insul., Vol. 27, No. 6, pp. 1152-1158, 1992.

[38] G. Mazzanti, G. C. Montanari, and L.A. Dissado, "Electrical aging and life models: the role of space charge", IEEE Trans. Dielectr. Electr. Insul., Vol. 12, pp. 876-890, 2005.

[39] E. Ildstad, J. Sletbak, B.R. Nyberg, and J.E. Larsen, "Factors affecting the choice of insulation system for extruded HVDC Power Cables", paper D1-203, CIGRE Session 2004.

[40] P. Morshuis, and M. Jeroense, "Space charge measurements on impregnated paper: a review of the PEA method and a discussion of results”, IEEE Electr. Insul. Mag., Vol. 13, No. 3, pp. 26-35, 1997.

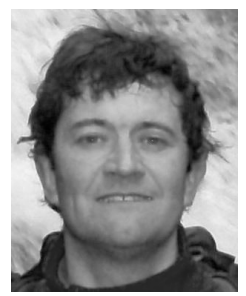

Giovanni Mazzanti (M'04) is an Associate Professor of HV Engineering and Power Quality at the University of Bologna, Italy. His interests are life modeling, reliability and diagnostics of $\mathrm{HV}$ insulation, power quality, renewables and human exposure to electro-magnetic fields. Since 2009 he is consultant of TERNA (the Italian TSO) in the HVDC and HVAC cable systems area. He is author or coauthor of more than 200 published papers, and coauthor of the book HVDC Extruded Cable Systems: Advances in Research and Development, Wiley-IEEE Press July 2013. He is chairman of the IEEE DEIS TC "HVDC Cable Systems".

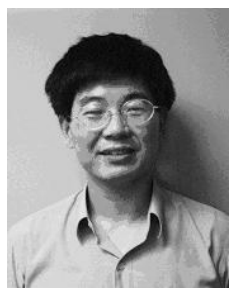

George Chen George Chen (SM'11) was born in China in 1961. He received the B.Eng. (1983) and M.Sc. (1986) degrees in electrical engineering from Xi'an Jiaotong University, China. After he obtained the Ph.D. degree (1990) from the University of Strathclyde, UK, on the work of permanent changes in electrical properties of irradiated low-density polyethylene, he joined the University of Southampton as postdoctoral research fellow and became a senior research fellow subsequently. In 1997 he was appointed as a research lecturer and promoted to a Reader in 2002 . He is now the professor of high voltage engineering at the University of Southampton and a visiting professor of Xi'an Jiaotong University. Over the years, he has developed a wide range of interests in high voltage engineering and electrical properties of materials and published over 300 papers. He is active in the IEEE DEIS TC "HVDC Cable Systems".

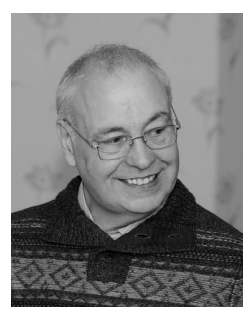

John C. Fothergill (SM'95-F'04) was born in Malta in 1953. He graduated from the University of Wales, Bangor, in 1975 with a Batchelor's degree in electronics. He continued at the same institution, working with Pethig and Lewis, gaining a Master's degree in Electrical Materials and Devices in 1976 and doctorate in the Electronic Properties of Biopolymers in 1979. Following this he worked as a senior research engineer leading research in electrical power cables at STL, Harlow, UK. In 1984 he moved to the University of Leicester where he later gained a personal chair in Engineering In 2012 he moved to City University London where he is Pro Vice-Chancellor (Research and Enterprise). He is active in the IEEE DEIS TC "HVDC Cable Systems". 


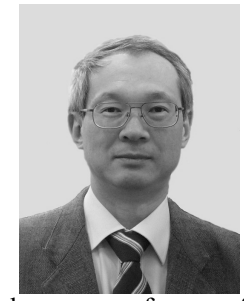

Naohiro Hozumi (M'90) Naohiro Hozumi was born in Kyoto, Japan on 2 April 1957. He received his B.S., M.S. and Ph.D. degrees in 1981, 1983 and 1990 from Waseda University. He was engaged in Central Research Institute of Electric Power Industry (CRIEPI) from 1983 to 1999 . He was an associate professor of Toyohashi University of Technology from 1999 to 2006, and a professor of Aichi Institute of Technology from 2006 to 2011. Since 2011, he has been a professor of Toyohashi University of Technology. He has been engaged in the research in insulating materials and diagnosis for high voltage equipment, acoustic measurement for biological and medical applications, etc. He was awarded in 1990 and 1999 from IEE of Japan for his outstanding research papers. He is a member of IEEE, IEE of Japan and the Acoustic Society of Japan. He is active in the IEEE DEIS TC "HVDC Cable Systems".

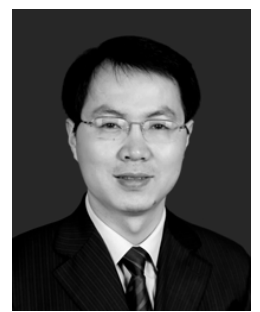

Jian Li (M'05-SM'11) received the M.S. and Ph.D. degrees in electrical engineering in 1997 and 2001, respectively from Chongqing University, Chongqing, China. He is currently a professor and the head of High Voltage and Insulation Technology Department at Chongqing University. His major research interests include online detection of insulation condition in electrical devices, partial discharges, and insulation fault diagnosis for high voltage equipment, environment-friendly liquid dielectrics, anti-icing coating of insulator and transmission line, and polyethylene/organic-montmorillonite nano-composites. He is an author and coauthor of more than 50 journal papers and 50 papers published in proceedings of international conferences. He is active in the IEEE DEIS TC "HVDC Cable Systems".

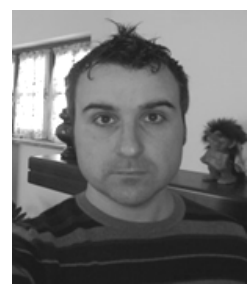

Massimo Marzinotto (S'97, M'01, SM'09) received the Master degree and the Ph.D. degree in electrical engineering at "La Sapienza" University of Roma in 2000 and 2006, respectively. From 2001 to 2008 he joined the Electrical Engineering Department - "La Sapienza" University of Roma. Since 2008 he joined TERNA (the Italian TSO). His main interests are high polymeric materials, power cables, insulators, applied statistics, insulation coordination and transients. He is member of IEEE-DEIS, IEEE-PES, CIGRE, CEI (Italian Electrotechnical Committee) and he is active in different IEEE Committees and CIGRE WGs. He is author and co-author of different international publications on IEEE transactions and conferences, and coauthor of the book HVDC Extruded Cable Systems: Advances in Research and Development, Wiley-IEEE Press, 2013. He is active in the IEEE DEIS TC "HVDC Cable Systems" and "Outdoor Insulation".

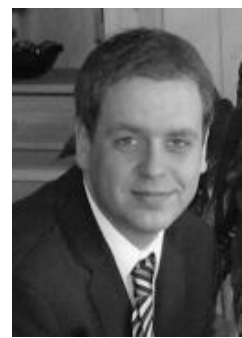

Frank Mauseth (M'11) received his M.Sc. degree in electrical engineering from Delft University of Technology, The Netherlands, in 2001. Since then he has been with the Norwegian University of Science and Technology (NTNU) in Trondheim, Norway, where he also received his Ph.D. degree in 2007 and now is working as an Associate Professor at NTNU. Main fields of interest are high voltage insulation materials and systems, measurement methods and testing. He is active in the IEEE DEIS TC "HVDC Cable Systems".

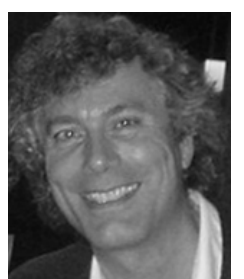

Peter Morshuis (M'95) received the Master degree in electrical engineering from Delft University of Technology. Since 1988, he has been a staff member of the High Voltage Group at Delft University of Technology where he was awarded the Ph.D. degree in electrical engineering in 1993. In 1998, he was a visiting Professor at the University of Bologna. Since 1999, he is an Associate Professor in High Voltage Engineering at Delft University of Technology and he is involved in teaching M.Sc. students in the field of high voltage. In 2012, he started Solid Dielectric Solutions, a company focusing on training/education and consultancy in the field of dielectrics and electrical insulation. His most important fields of interest are HVDC (materials and systems), space charge, partial discharge, aging of electrical insulation and on-line monitoring of high voltage equipment. He is involved in a number of CIGRÉ and IEEE activities. $\mathrm{He}$ is an Associate Editor of the IEEE Transactions on Dielectrics and Electrical Insulation and he is active in the IEEE DEIS TC "HVDC Cable Systems".

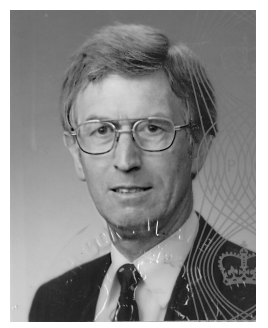

Clive W Reed (M'80) graduated from the University of Bristol, England, in 1956 with a first class special honors BSc degree in chemistry and with a Ph.D. degree in physical chemistry in 1960 . After a post-doctoral fellowship in low temperature physics at the National Research Council of Canada in Ottawa, in 1963 he joined GE Corporate R\&D, Schenectady, NY where he worked on HV insulation materials and design for numerous commercial applications until retirement in 2002 . He is past chairman of the NAS/NRC Washington DC Conference on Electrical Insulation and Dielectric Phenomena, the NAS/NRC Committee on Dielectrics, and CIGRE's Study Committee on Materials for Electrotechnology. His awards include the 2011 Philip Sporn Award "for Cumulative Career Contributions to the Advancement of System Integration in the Theory, Design, and Operation of Large High voltage Electrical Systems in the United States". Presently, he is an independent consultant on $\mathrm{HV}$ insulation materials and design. He is active in the IEEE DEIS TC "HVDC Cable Systems".

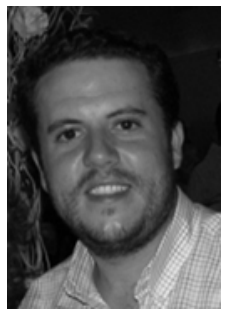

Antonios Tzimas (M'08) received the B.Eng. (2001) and M.Sc. (2003) degrees in electrical and electronic engineering from the University of Leicester where after completing his national service duties in Greece he returned to study for the $\mathrm{Ph} . \mathrm{D}$. degree on ageing properties of cross linked polyethylene. He received the degree in 2008. He then joined the National Grid High Voltage laboratory at the University of Manchester as Post-Doctoral researcher till 2013 working on the ageing of outdoor and cable insulation. Currently he is working at Alstom Grid in Materials and HV Technology Research center. In 2011 he became the chair of IEEE Dielectrics and Electrical Insulation Society GOLD membership and part of the society's membership committee. He is also board member of an annual Conference on Electrical Insulation and Dielectric Phenomena (CEIDP). He is active in the IEEE DEIS TC "HVDC Cable Systems".

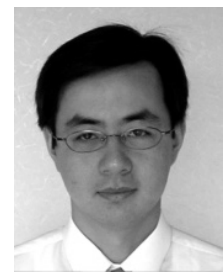

Kai Wu (M'99-SM'13) received the M.S. and Ph.D. degrees in electrical engineering from Xi'an Jiaotong University, China in 1992 and 1998, respectively. He was a postdoctoral fellow from 1998 to 2000 and then joined the staff from 2000 to 2003 at Nagoya University, Japan. In 2003, he worked as a research associate at the University of Leicester, UK. In 2004 and 2005, he was a visiting researcher at the Central Research Institute of Electric Power Industry, Japan. Since 2006, he has become a Professor of Xi'an Jiaotong University, China. $\mathrm{He}$ is active in the IEEE DEIS TC "HVDC Cable Systems". 